Mens

Revue d'histoire intellectuelle et culturelle

mens

\title{
Le journal régional, organe politique et outil de développement : l'exemple du Franco-Canadien et du Canada français de Saint-Jean
}

\section{Dominique Marquis}

Volume 17, numéro 1-2, automne 2016, printemps 2017

S’organiser, se distinguer, se donner une identité : vie culturelle et sociabilités en région au Québec $\left(\mathrm{XIX}^{\mathrm{e}}-\mathrm{XX}^{\mathrm{e}}\right.$ siècles)

URI : https://id.erudit.org/iderudit/1050784ar

DOI : https://doi.org/10.7202/1050784ar

Aller au sommaire du numéro

Éditeur(s)

Centre de recherche en civilisation canadienne-française

ISSN

1492-8647 (imprimé)

1927-9299 (numérique)

Découvrir la revue

Citer cet article

Marquis, D. (2016). Le journal régional, organe politique et outil de développement : l'exemple du Franco-Canadien et du Canada français de Saint-Jean. Mens, 17(1-2), 79-106. https://doi.org/10.7202/1050784ar
Résumé de l'article

Fondé en 1860, Le Franco-Canadien est d'abord l'outil politique de Félix-Gabriel Marchand. À la suite de tractations, le journal échappe à son influence et, en 1893, Le Canada français apparaît dans le paysage de Saint-Jean pour assurer une voix au Parti libéral du Québec. Deux ans plus tard, les deux journaux fusionnent et Le Canada français devient à la fois un organe politique et un outil de développement régional. Cet article porte sur l'évolution de la pratique journalistique du Franco-Canadien / Canada français de 1860 à 1970. Il montre comment, alors qu'apparaissent de nouveaux modes de production et de diffusion de la presse, le journal régional doit s'adapter à de nouvelles réalités médiatiques afin d'assurer sa survie. La longévité exemplaire du Canada français montre que ce journal a su tirer son épingle du jeu et offrir à ses lecteurs un produit aussi attrayant que les journaux montréalais, mais distinct par son contenu régional. 


\title{
Le journal régional, organe politique et outil de développement : l'exemple du Franco-Canadien et du Canada français de Saint-Jean
}

\author{
Dominique Marquis \\ Université du Québec à Montréal
}

\section{Résumé}

Fondé en 1860, Le Franco-Canadien est d'abord l'outil politique de Félix-Gabriel Marchand. À la suite de tractations, le journal échappe à son influence et, en 1893, Le Canada français apparaît dans le paysage de Saint-Jean pour assurer une voix au Parti libéral du Québec. Deux ans plus tard, les deux journaux fusionnent et $\mathrm{Le}$ Canada français devient à la fois un organe politique et un outil de développement régional. Cet article porte sur l'évolution de la pratique journalistique du Franco-Canadien / Canada français de 1860 à 1970. Il montre comment, alors qu'apparaissent de nouveaux modes de production et de diffusion de la presse, le journal régional doit s'adapter à de nouvelles réalités médiatiques afin d'assurer sa survie. La longévité exemplaire du Canada français montre que ce journal a su tirer son épingle du jeu et offrir à ses lecteurs un produit aussi attrayant que les journaux montréalais, mais distinct par son contenu régional.

\section{Abstract}

Founded in 1860, Le Franco-Canadien is first and foremost the political tool of Félix-Gabriel Marchand. As a result of negotiations, the newspaper escapes its influence and, in 1893, Le Canada français appears in the 
landscape of Saint-Jean to ensure a voice for the Liberal Party of Quebec. Two years later, the two newspapers merge and Le Canada français becomes both a political body and a tool for regional development. This article focuses on the evolution of the journalistic practice of FrancoCanadien/Canada français from 1860 to 1970. It shows how, while new modes of production and diffusion of the press appear, the regional newspaper must adapt to new media realities to ensure its survival. The exemplary longevity of Le Canada français shows that this newspaper has managed to stand out and offer its readers a product as attractive as the Montreal newspapers, but distinct in its regional content.

Au Québec, les historiens s’intéressent depuis longtemps au contenu des journaux puisqu'ils y puisent régulièrement leur matière première. En utilisant souvent des méthodes plus ou moins sophistiquées d'analyse de contenu et de discours, plusieurs travaux ont scruté les idées et les représentations véhiculées dans les journaux ${ }^{1}$. Nombreux sont les exemples de ces analyses $\mathrm{du}$ " discours de la presse sur... ${ }^{2} »$. Dans tous ces travaux, le journal a été davantage considéré comme une archive que comme un corpus pouvant être étudié en tant que tel. Ces monographies ont certes souligné l'éventail très varié de la presse comme agent de diffusion des idées ou comme outil de représentation; toutefois, ces études ne peuvent pas être associées à une histoire de la presse.

${ }^{1}$ Il en a résulté, entre autres, le volumineux travail de Fernand Dumont, Les idéologies au Canada français, Québec, Les Presses de l'Université Laval, 5 vol., 1969-1981, ou encore des travaux portant sur des individus. Voir, par exemple : Marie-Ève Tanguay, La pensée d'Edmond Turcotte, éditorialiste au journal Le Canada (19311937), mémoire de maîtrise (histoire), Montréal, Université de Montréal, 2008; ou Pierre Anctil, Fais ce que dois : 60 éditoriaux pour comprendre Le Devoir sous Henri Bourassa, 1910-1932, Québec, Éditions du Septentrion, 2010.

${ }^{2}$ Voir, par exemple : François Zombecki, La perception de la fête internationale des travailleurs dans les journaux canadiens entre 1906 et 1945, mémoire de maîtrise (histoire), Montréal, Université du Québec à Montréal, 2008; ou Pierre-Luc Beauchamp, Le sport et l'identité collective au Canada: la série du siècle de 1972, mémoire de maîtrise (histoire), Montréal, Université du Québec à Montréal, 2005. 


\section{Une historiographie en développement}

Des historiens ont cependant développé une approche qui conçoit la presse comme un objet d'étude plutôt que comme un véhicule idéologique. Ainsi, les importants travaux de Jean de Bonville ont analysé le passage de la presse d'opinion à la presse d'information et ont suscité de nombreuses et précieuses réflexions sur le rôle socioéconomique des journaux ${ }^{3}$. Quelques individus marquants du monde de la presse, propriétaires, imprimeurs ou rédacteurs, ont reçu une attention particulière de la part des chercheurs qui ont voulu comprendre les liens entre le journalisme et la vie publique ${ }^{4}$. La parole des femmes a aussi, et fait toujours, l'objet de recherches approfondies : on s'est notamment intéressé à la place qu'elles ont réussi, tant bien que mal, à se tailler dans la presse, autant comme actrices que comme sujets 5 .

Plusieurs grands quotidiens nationaux ont déjà fait l'objet de monographies : Le Devoir a été analysé en profondeur ${ }^{6}$. Une étude récente du quotidien Montréal-Matin a souligné les relations particulières entre ce journal aux allures très populaires, voire populistes, et un parti politique ${ }^{7}$. En mettant l'accent sur la pratique journalistique, j'ai aussi cherché à comprendre comment un quotidien

3 Jean de Bonville, La presse québécoise de 1884 à 1914 : genèse d'un média de masse, Québec, Les Presses de l'Université Laval, 1988.

4 Voir, par exemple, Réal Bélanger, Henri Bourassa : le fascinant destin d'un homme libre (1868-1914), Québec, Les Presses de l'Université Laval, 2013; ou Hélène Pelletier-Baillargeon, Olivar Asselin et son temps, Montréal, Éditions Fides, 3 vol., 1996, 2001 et 2010.

5 Kay Rex, No Daughter of Mine: The Women and History of the Canadian Women's Press Club, 1904-1971, Toronto, University of Toronto Press, 1995; Josette Brun (dir.), Interrelations femmes-médias dans l'Amérique française, Québec, Les Presses de l'Université Laval, 2009.

6 Plusieurs travaux portant sur Le Devoir ont été réalisés. Voir, entre autres, le numéro spécial de la revue Communications consacré au centenaire du journal, vol. 29, $\mathrm{n}^{\circ} 2$ (2012), ou l'ouvrage dirigé par Robert Lahaise, Le Devoir, reflet du Québec au $20^{e}$ siècle, LaSalle, Hurtubise HMH, 1994.

7 Mathieu Noël, Le Montréal-Matin (1930-1978), un journal d'information populaire, thèse de doctorat (histoire), Montréal, Université du Québec à Montréal, 2014. 
d'information comme L'Action catholique a pu servir les intérêts de l'Église, tout en respectant les règles inhérentes à ce type de presse ${ }^{8}$. La Presse attend toujours son "biographe " même si une synthèse destinée à faire connaître son histoire au grand public a été publiée en 1984 à l'occasion de son centième anniversaire. Ces exemples ne doivent pas faire oublier que l'histoire des entreprises de presse est encore peu développée au Québec; on préfère analyser la presse à partir d'autres prémisses.

Ainsi, plusieurs études ont inclus la presse périodique, journaux et revues, dans une analyse plus globale du développement de la vie culturelle au Québec et plus particulièrement à Montréal ${ }^{9}$. La presse devient ainsi non seulement porteuse de discours, mais aussi un élément structurant de la vie culturelle des $\mathrm{XIX}^{\mathrm{e}}$ et $\mathrm{XX}^{\mathrm{e}}$ siècles. Le concept de culture médiatique, qui englobe les journaux - et tous les médias - dans un vaste système de représentation, est d'ailleurs très utile pour comprendre ce rôle structurant de la presse ${ }^{10}$. Tous ces travaux contribuent à enrichir l'historiographie de la presse en allant au-delà de son contenu idéologique ou informatif. L'interdisciplinarité est aussi au rendez-vous; ces études sont l'œuvre non seulement d'historiens, mais aussi de littéraires, d'historiens de l'art ou de spécialistes des sciences de l'information et des communications qui apportent tous à ce champ des perspectives analytiques différentes. L'histoire de la presse québécoise prend donc différentes orientations,

8 Dominique Marquis, Un quotidien pour l'Église : L'Action catholique, 1910-1940, Montréal, Leméac éditeur, 2004.

9 Voir, par exemple, Chantal Savoie, "La page féminine des grands quotidiens montréalais comme lieu de sociabilité littéraire au tournant du $\mathrm{xx}^{\mathrm{e}}$ siècle ", Tangence, $\mathrm{n}^{\circ} 80$ (hiver 2006), p. 125-142, ou l'important projet de recherche dirigé par Micheline Cambron "La presse montréalaise de l'entre-deux guerres, lieu de transformation de la vie culturelle et de l'espace public ».

${ }^{10}$ Ce concept a surtout été utilisé par les littéraires, mais il s'avère aussi très pertinent pour comprendre l'évolution du rôle des médias dans la société. Concernant la genèse de ce concept, voir Pascal Durand, "La culture médiatique au XIX ${ }^{\text {ème }}$ siècle : essai de définition-périodisation ", Quaderni, no 39 (automne 1999), p. 29-40. Voir aussi Guillaume Pinson, La culture médiatique francophone: Europe-Amérique du Nord 1760-1930, Québec, Les Presses de l'Université Laval, 2016. 
mais quelle place est accordée à la presse régionale dans ce champ historiographique? Comment les études qui s'y sont consacrées ontelles appréhendé cet objet?

\section{La presse régionale, le parent pauvre de l'historiographie québécoise}

Malheureusement, force est de constater que les journaux régionaux, quotidiens ou hebdomadaires, sont les parents pauvres de la recherche en histoire de la presse québécoise. Comme je l'ai déjà mentionné, les analyses portant sur les entreprises de presse sont plutôt rares, mais les journaux de Montréal et de Québec ont été beaucoup plus utilisés et analysés que leurs homologues régionaux. Une rare étude d'un appareil de presse régional, celui de la ville de Trois-Rivières, a souligné non seulement la richesse du corpus trifluvien, mais aussi le fait que cette presse régionale est toujours un peu à la remorque de la grande presse d'information ${ }^{11}$. Cette recherche ne peut toutefois être considérée que comme une mise en bouche; il faudrait l'approfondir davantage à la lumière de données plus complètes.

À la fin des années 1980, l'Institut québécois de recherche sur la culture, devenu l'INRS, a commencé la production d'une série d'ouvrages de synthèse sur l'histoire des régions du Québec, entreprise majeure achevée avec la région du Centre-du-Québec en 2013. Dans chacun de ces volumineux ouvrages, une partie a été réservée à la vie culturelle; c'est dans cette section qu'a été soulignée l'action des journaux dans le développement économique et culturel des régions. Le traitement accordé à l'histoire de la presse dans ces synthèses est très variable et dépend beaucoup des champs d'intérêt des collaborateurs et de la vigueur des périodiques de chaque région. On y énumère les principaux titres, du moins ceux qui ont connu une importante longévité; on souligne le rôle que ces journaux ont joué dans le développement régional; on mentionne le nom de journalistes

11 Mélanie Couture, L'appareil de presse trifluvien entre 1852 et 1920, mémoire de maîtrise (histoire), Montréal, Université du Québec à Montréal, 2008. 
qui, souvent, ont commencé leur carrière en région avant de connaitre le succès dans les grands centres urbains. Même si la portion accordée au développement de la presse régionale y est parfois congrue, ces synthèses ont néanmoins le mérite d'indiquer aux chercheurs que partout au Québec, la presse écrite a été un élément important de la promotion culturelle et économique des régions, qu'elle a connu son âge d'or durant la première moitié du $\mathrm{xx}^{\mathrm{e}}$ siècle et que le mouvement de concentration de la presse, qui s'est intensifié au Québec à partir des années 1970, a nui à l'expression écrite de ces régionalismes.

Dans le but de faire mieux connaître la presse régionale, Hebdos Québec, une association qui regroupe 150 journaux hebdomadaires francophones, a, entre 2008 et 2010, publié neuf petits volumes relatant l'histoire de l'association et des hebdomadaires régionaux du Québec. Ces ouvrages destinés au grand public veulent surtout souligner le travail des bâtisseurs de ces entreprises; les collaborateurs ont entrepris des recherches dans les archives et les journaux locaux et ils ont mis en lumière les motivations des pionniers de la presse régionale. Ils ont aussi retrouvé les acteurs qui ont participé à l'expansion de la presse hebdomadaire tout en contribuant au développement régional. Ces ouvrages ne proposent pas une analyse exhaustive des hebdomadaires, mais ils permettent de prendre la mesure de la richesse de ce corpus ${ }^{12}$.

D'autres exemples pourraient s'ajouter ${ }^{13}$, mais ils sont si peu nombreux qu'il est possible de conclure ici que la recherche sur la presse régionale québécoise mérite manifestement d'être enrichie. Plusieurs perspectives de recherche s'ouvrent aux chercheurs, mais il serait souhaitable que ces travaux ne considèrent plus uniquement les journaux comme des archives permettant d'éclairer l'évolution politique, économique ou sociale d'une région, mais comme des

12 Jean-Pierre Malo (coordonnateur), Histoire de la presse hebdomadaire au Québec, Montréal, Hebdos Québec, 9 vol., 2008-2010.

13 Notamment le travail pionnier de Jean-Pierre Kesteman, "Le Progrès " (1874-1878): étude d'un journal de Sherbrooke, Groupe de recherche en histoire des Cantons de l'Est, Département d'histoire, Sherbrooke, Université de Sherbrooke, 1979. 
outils qui ont participé activement à cette évolution et comme des corpus qui ont eu une vie propre.

Un problème doit néanmoins être soulevé : celui de l'accessibilité de ces corpus et de la conservation de ces journaux. En ce qui concerne les archives, de nombreux fonds produits par les entreprises propriétaires de journaux aujourd'hui disparues ont été détruits ou dispersés. Certains fonds privés d'individus permettent parfois de dévoiler des pans de cette histoire, mais ils sont aussi très dispersés. Il est donc difficile d'accéder au cœur de la vie de ces journaux.

Par contre, le vaste projet de numérisation du patrimoine documentaire du Québec lancé par Bibliothèque et Archives nationales du Québec (BAnQ) il y a plusieurs années vient pallier cette accessibilité réduite. L'intention est plus que louable, et il est maintenant possible de consulter en ligne plus de 275 périodiques québécois, provenant de toutes les régions et couvrant toutes les périodes. L'historien de la presse a désormais accès à un large éventail de périodiques. Il ne lui reste plus qu'à faire parler ces corpus.

Les journaux régionaux développent-ils des stratégies de communication qui leur sont propres? Comment se distinguent-ils des grands journaux nationaux qui leur livrent sans aucun doute une féroce concurrence? Comment attirer et conserver un lectorat qui est très courtisé par ces journaux nationaux? Voilà quelques questions auxquelles je souhaiterais répondre dans cet article à partir de l'exemple d'un journal régional singulier par sa longévité, qui a célébré, en 2015, son $155^{\mathrm{e}}$ anniversaire, Le Franco-Canadien/ Canada français de Saint-Jean (aujourd'hui Saint-Jean-sur-Richelieu). L'analyse de la pratique journalistique du Franco-Canadien puis du Canada français permettra de mesurer comment ces journaux régionaux modulent leur action pour assurer leur survie à l'intérieur d'un système sur lequel ils n'ont que très peu de prise. L'exercice proposé ici veut aussi montrer tout ce qu'une lecture fine des journaux et un regard attentif sur leur matérialité peuvent dévoiler sur l'histoire d'un organe de presse. 


\section{LE FRANCD-CANADIEN,} ORGANE: DU DISTRICT D'IBERVILLE.

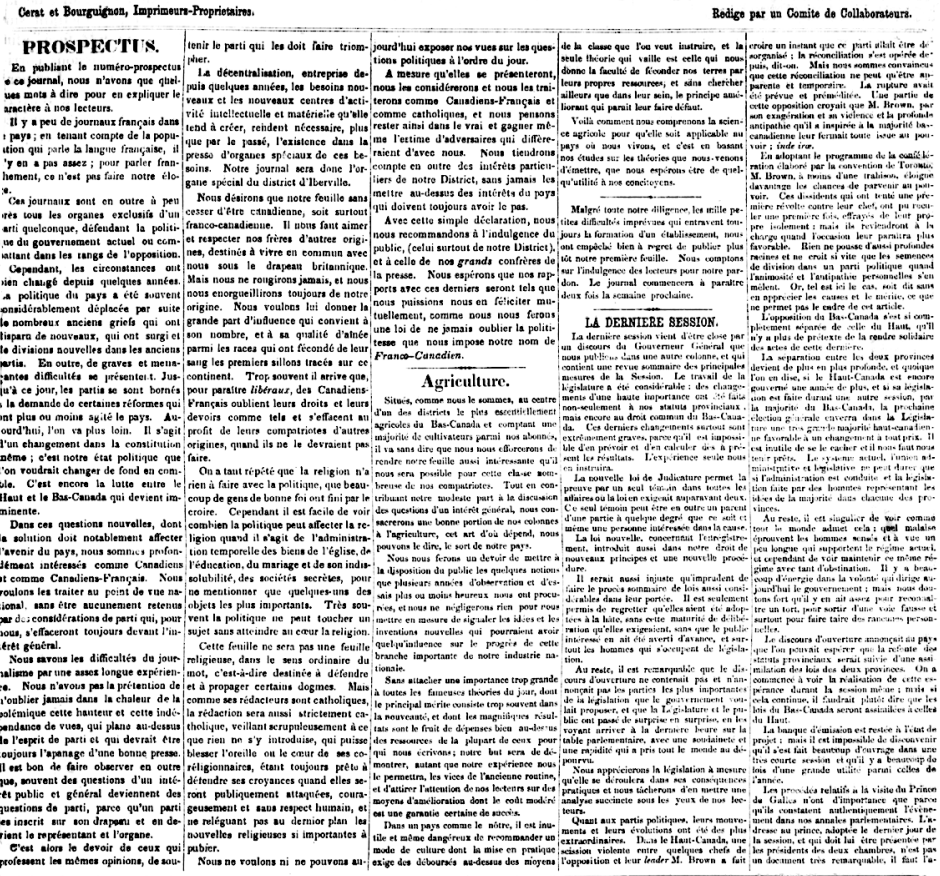

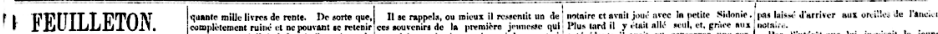

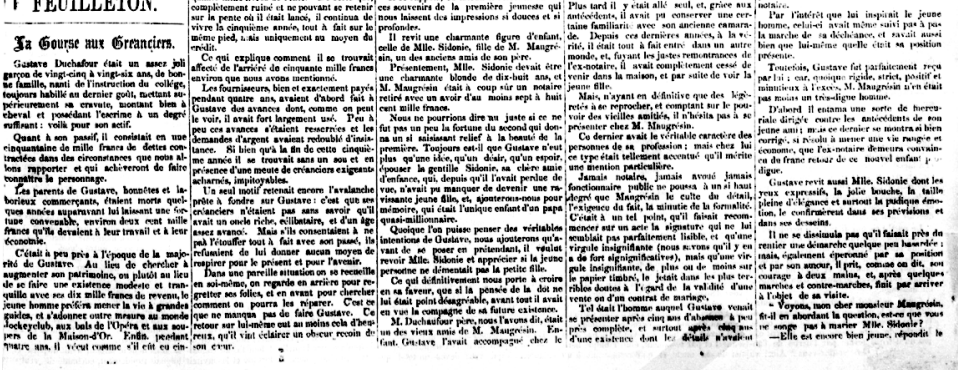

Figure 1 - Le Franco-Canadien, $1^{\text {er }}$ juin 1860, p. 1.

Source: Bibliothèque et Archives nationales du Québec, [En ligne], [http://numerique. banq.qc.ca/patrimoine/details/52327/1792224]. 


\section{Le Franco-Canadien, un hebdomadaire régional au cour des débats politiques}

Durant la deuxième moitié du XIX ${ }^{\mathrm{e}}$ siècle, le développement des réseaux de transport ferroviaire favorise la croissance économique des régions, notamment celles qui sont situées en périphérie de Montréal. Au cours de cette période, la Montérégie est témoin d'une expansion importante : les progrès dans les domaines agricole et industriel contribuent à sa prospérité. Expansion du réseau ferroviaire, croissance industrielle, prospérité agricole, urbanisation : les ingrédients de base sont rassemblés pour assurer la survie d'un journal. Le Courrier de Saint-Hyacinthe, aujourd'hui le plus ancien hebdomadaire francophone en Amérique, est fondé en 1853.

La municipalité de Saint-Jean n'est pas en reste : elle a reçu le statut de ville en 1856 et elle est déjà un carrefour commercial important, maritime par la voie fluviale du Richelieu, qui mène aux États de la Nouvelle-Angleterre, et ferroviaire grâce au premier chemin de fer en sol canadien reliant Saint-Jean à La Prairie et inauguré en 1836. Là aussi, les conditions économiques sont réunies pour soutenir un journal, conditions auxquelles s'ajoutent des enjeux politiques qui soulèvent les passions. Le $1^{\text {er }}$ juin 1860, Le Franco-Canadien est lancé.

Ce bihebdomadaire de quatre pages est désigné comme étant la propriété de deux imprimeurs déjà actifs dans la région, Pierre Cérat et Isaac Bourguignon. Dès le 17 juillet, cependant, seul le nom de Bourguignon est mentionné comme imprimeur-propriétaire. Ces hommes n'étant pas journalistes, la une indique qu'il est rédigé par un "Comité de collaborateurs » : les avocats Charles-Joseph Laberge et Alfred-Napoléon Charland, et le notaire Félix-Gabriel Marchand y publient des textes. Sans pouvoir le prouver hors de tout doute, Marchand en est certainement le principal rédacteur. Très engagé dans sa communauté, il sera élu député libéral à l'Assemblée législative du Québec en 1867 et il occupera le poste de premier ministre de la 
province de Québec de 1897 jusqu'à son décès en $1900^{14}$. Il est important de préciser que la circonscription électorale de Saint-Jean élira sans exception un candidat libéral aux élections provinciales jusqu'en 1941 et il en sera de même aux élections fédérales jusque dans les années $1960^{15}$. Le Franco-Canadien, qui affichera bientôt ses couleurs libérales, s'implante donc dans un sol très fertile et réceptif aux idées libérales.

Le prospectus du journal donne une bonne indication des objectifs du Franco-Canadien, et ses couleurs sont clairement annoncées. Marchand s'oppose au projet de confédération qui fait alors l'objet de discussions très intenses et il entend bien faire connaître sa position par la voix de son journal. Ce dernier ne sera toutefois pas le porteétendard d'un parti, les questions d'actualité seront traitées « au point de vue national, sans être aucunement retenues par des considérations de $\operatorname{parti}^{16}$ ». Mais l'heure est néanmoins grave et les enjeux liés au projet d'une nouvelle constitution doivent être discutés. Le journal veut aussi combler une lacune : il n'y a pas suffisamment de journaux francophones dans ce " pays".

Le nouveau périodique aura tous les attributs d'une bonne presse : hauteur et indépendance de vues seront au rendez-vous. Il sera catholique, mais pas religieux, donc pas dogmatique; il sera nationaliste, c'est-à-dire que tout en aimant et respectant " nos frères d'autres origines ${ }^{17}$ ", les rédacteurs seront toujours fiers de leur origine

${ }^{14}$ Michèle Brassard et Jean Hamelin, " Marchand, Félix-Gabriel », dans Dictionnaire biographique du Canada en ligne (ci-après $D B C$ ), vol. 12, Québec, Université Laval; Toronto, Université de Toronto, [http://www.biographi.ca/fr/bio/marchand_ felix_gabriel_12F.html].

15 En 1941, un candidat de l'Union nationale a remporté le siège de Saint-Jean ("Les résultats électoraux depuis 1867, Saint-Jean à Saint-Sauveur", sur le site de l'Assemblée nationale du Québec, [http://www.assnat.qc.ca/fr/patrimoine/ resultatselec/s2.html] et "Historique des circonscriptions depuis 1867 ", sur le site du Parlement du Canada, [https://lop.parl.ca/About/Parliament/ FederalRidingsHistory/hfer.asp? canName $=\&$ canParty $=131 \&$ Language $=$ F \& rid Name $=\&$ ridProvince $=0 \&$ Search $=$ Cres\&submit $1=$ Search $]$.

16 "Prospectus », Le Franco-Canadien, $1^{\mathrm{er}}$ juin 1860, p. 1.

17 Lire ici les Anglais. 
française. Le journal fait passer le pays avant la région : " Nous tiendrons compte en outre des intérêts particuliers de notre District, sans jamais les mettre au-dessus des intérêts du pays qui doivent toujours avoir le pas. " On demande finalement l'indulgence du public et, dans un contexte où des journaux montréalais sont déjà diffusés dans la région, celle de ses " grands confrères de la presse ${ }^{18}$ ».

Les rédacteurs adoptent une pratique journalistique fréquemment en usage à l'époque. La mise en page est dense, composée sur cinq colonnes, sans gros titres ni manchettes. La une propose les nouvelles politiques de la session parlementaire, lesquelles se poursuivent à la page deux. Un feuilleton, La course aux créanciers, une nouvelle de Charles Grandvallet parue initialement dans le Journal pour tous de Paris aux environs de 1858, débute en rez-de-chaussée de la une et se poursuit à la page suivante ${ }^{19}$. Malgré l'importance accordée aux nouvelles politiques, le journal n'est pas exempt de faits divers. Par exemple, le récit d'un « horrible » meurtre et du procès qui en découle est présenté et, semaine après semaine, le lecteur peut suivre le fil des événements presque comme un feuilleton. Des nouvelles internationales sont proposées, mais principalement en brèves et par la voie du fil télégraphique. Une longue liste des lois adoptées par le gouvernement complète la partie informative de ce premier numéro. Finalement, les deux dernières pages sont consacrées à des publicités, provenant presque exclusivement de commerces et de représentants des professions libérales de la municipalité de Saint-Jean. Au départ, l'ancrage régional de ce journal émane essentiellement de la publicité.

En 1860, nous avons donc affaire à un journal de facture plutôt classique, lancé à l'instigation d'un homme qui a des ambitions politiques et qui, alors que d'importants débats ont lieu sur la scène politique, veut faire valoir son point de vue auprès de ses concitoyens.

18 "Prospectus ", Le Franco-Canadien, $1^{\mathrm{er}}$ juin 1860, p. 1. En italique dans le texte

19 La mise en page varie beaucoup d'un journal à l'autre, mais il est alors très fréquent d'insérer le feuilleton à la une, parfois en rez-de-chaussée, parfois à la verticale sur les colonnes de droite, comme le pratique La Minerve de Montréal. 
Ne serait-ce la nature et la provenance des publicités, ce journal ressemblerait à la plupart des journaux d'opinion publiés un peu partout au Québec. Mais l'histoire de cette entreprise est assez singulière et elle mérite d'être approfondie parce qu'elle est un exemple des relations complexes qui peuvent lier les imprimeurs, les propriétaires de journaux et les rédacteurs.

En effet, en mars 1867, Félix-Gabriel Marchand devient propriétaire du Franco-Canadien afin, dit-il, de redresser une situation qui devenait de plus en plus difficile et d'assurer que la région aura toujours son organe de presse francophone ${ }^{20}$. "Il nous a donc paru urgent de mettre la main à l'œuvre et d'essayer, dans la faible mesure de nos forces, de communiquer une nouvelle vigueur au seul organe que possède la population française dans cette partie du pays ${ }^{21}$. " Le nouveau propriétaire affiche aussi plus clairement sa couleur politique, libérale, mais il agit ainsi par nécessité et il se défend bien de s'enliser dans la partisanerie ${ }^{22}$. «[I]l est impossible que l'on s'occupe activement d'affaires publiques sans se rattacher à l'un des partis qui se partagent l'opinion. Vouloir le contraire serait se condamner soi-même à l'impuissance ou, pour mieux dire, à l'insignifiance, et compromettre le succès des projets dont on peut reconnaître l'utilité et que l'on désire réaliser ${ }^{23}$. » Il se fait toutefois rassurant : les discussions seront fermes, mais polies, toute attaque personnelle sera exclue des pages du journal. Se positionnant toujours en relation avec les grands journaux montréalais, il promet aussi que " le Franco-Canadien sera à l'avenir aussi promptement et aussi exactement renseigné que les meilleurs journaux de nos grandes villes $^{24}$ " grâce à des ententes qui facilitent les échanges d'abonnement avec des journaux européens et américains. Marchand ne demeure cependant propriétaire du journal que jusqu'en 1877, alors que le

20 Bourguignon est alors identifié comme administrateur.

21 Le Franco-Canadien, $1^{\mathrm{er}}$ mars 1867, p. 2.

${ }^{22}$ La lutte contre le projet de Confédération ayant été perdue, Marchand s'identifie dès lors plus clairement au Parti libéral.

23 Le Franco-Canadien, $1^{\text {er }}$ mars 1867, p. 2.

24 Ibid. 
cartouche d'entête du $1^{\text {er }}$ juin 1877 précise qu'Isaac Bourguignon est à nouveau propriétaire et Marchand, rédacteur en chef $^{25}$. Les activités politiques de ce dernier prenant de plus en plus d'importance, il abandonne, sans tambour ni trompette, son titre de rédacteur en chef du Franco-Canadien en mars $1878^{26}$. Le journal demeure toutefois, sans contredit, l'outil politique de Marchand.

En septembre 1883, le journal, dont le rédacteur en chef est alors Gabriel Marchand, le fils de Félix-Gabriel, subit des transformations majeures. Après avoir produit durant plusieurs années une publication bihebdomadaire puis trihebdomadaire, la direction recule et opte pour une périodicité hebdomadaire, dont les coûts de production sont moins élevés et qui sera conservée jusqu'à nos jours. Les lecteurs ne doivent toutefois pas se sentir lésés par ce choix. On sollicite leur bienveillance et on les invite à payer leur abonnement dans les plus brefs délais ${ }^{27}$. On annonce que le journal a été amélioré; si la périodicité a été réduite, le format a, quant à lui, été bonifié pour atteindre " la dimension des plus grandes publications de notre province " (on passe de six à huit colonnes, mais toujours sur quatre pages) et le contenu a été enrichi pour en faire un véritable journal des familles. Le feuilleton occupe toute la une, la publicité couvre $25 \%$ de la surface totale du journal, réparti sur deux pages, et fait essentiellement la promotion des marchands locaux, et le reste du journal s'intéresse surtout aux actualités politiques nationales et plus particulièrement aux interventions de Félix-Gabriel Marchand à

25 «Félix-Gabriel Marchand », sur le site de l'Assemblée nationale du Québec, [http:// www.assnat.qc.ca/fr/deputes/marchand-felix-gabriel-4317/biographie.html]. Des numéros manquants dans la collection de Bibliothèque et Archives nationales du Québec (BAnQ) pour l'année 1877 ne permettent pas de trouver, dans le journal, une explication à ce revirement.

26 Le 12 mars 1878, son nom était encore inscrit comme rédacteur en chef dans le cartouche d'entête, alors que le 15 mars son nom n'y apparaît plus. Le journal n'explique pas ce retrait, mais il est plausible que ses nombreuses activités publiques l'empêchent de prendre à temps plein la responsabilité de la rédaction du journal ou qu'il souhaite prendre de manière officielle une distance par rapport au journal.

27 "À nos abonnés ", Le Franco-Canadien, 7 septembre 1883, p. 2. 
l'Assemblée législative. Quelques nouvelles internationales se glissent ici et là dans le journal. Manifestement, dans cette version remaniée, la notion de journal de famille reste encore à peaufiner, et même si le contenant a été modifié, on peut sans hésitation parler d'une mouture qui s'inscrit dans la continuité.

En une vingtaine d'années, les propriétaires du journal ont donc testé différentes formules afin de conserver les lecteurs ou en attirer de nouveaux, mais l'hebdomadaire demeure dans le giron de FélixGabriel Marchand et il est toujours conçu principalement comme un outil de diffusion des idées, voire comme une arme politique opposée aux conservateurs.

\section{Le Canada français entre en scène}

En juillet 1893, le Parti conservateur profite des nouvelles difficultés financières du journal pour manœuvrer et en prendre le contrôle tout en s'assurant que Bourguignon en demeure le propriétaire. Marchand, alors chef de l'opposition libérale à Québec, lance immédiatement, avec son ami l'avocat Alphonse Morin, Le Canada français. Ce nouvel hebdomadaire veut conquérir le lectorat de la région et, surtout, continuer de faire entendre la voix des libéraux en affichant désormais, et sans équivoque, à la une, son lien avec le Parti libéral. La lutte entre les deux journaux est enclenchée. Les invectives sont nombreuses. À la fin du mois d'août 1895, Marchand et Morin ont toutefois gain de cause dans cette bataille. Ils profitent d'une clause du contrat initial de fondation du Franco-Canadien, qui donnait le privilège à Marchand, si Bourguignon voulait se départir du journal ou en cas d'insolvabilité, de reprendre possession du Franco-Canadien. Ce dernier est fusionné avec Le Canada français ${ }^{28}$. Du jour au lendemain Le Franco-Canadien disparaît, et Marchand annonce à ses lecteurs que les deux journaux se sont réconciliés et

${ }^{28}$ Félix-Gabriel Marchand évoque cette clause dans un article dans lequel il explique aux lecteurs du Franco-Canadien la nouvelle situation du journal ("Explication ", Le Canada français, 30 août 1895, p. 3). 
que Le Franco-Canadien " rentre dans la voie du devoir côte à côte dans les rangs libéraux avec son jeune et vaillant confrère, Le Canada français $^{29}$ ". Ce journal assurera donc de manière non équivoque la défense du Parti libéral. Ce n'est qu'en 1937 que Le Canada français cessera d'être officiellement associé au Parti libéral. Non seulement les éditoriaux favorisent toujours le parti, mais une grande partie des pages $\mathrm{du}$ journal sont aussi utilisées pour faire valoir les positions libérales. Ainsi les faits et gestes du premier ministre libéral Wilfrid Laurier sont scrutés à la loupe et applaudis; les assemblées partisanes sont rapportées avec enthousiasme; les prises de position du Parti conservateur sont dénoncées de manière véhémente. Malgré quelques nouvelles locales, qui proposent surtout des mondanités et des faits divers, Le Canada français est d'abord et avant tout un journal d'opinion qui gravite essentiellement autour d'un axe politique.

Le journal poursuit tout de même son évolution et apporte des modifications à sa présentation matérielle. Le 17 juin 1898, Gabriel Marchand, qui avait abandonné la pratique du journalisme au profit d'un emploi à la fonction publique à la fin des années 1880, revient derrière le pupitre à titre de rédacteur-propriétaire du Canada français. Il amorce des changements matériels importants et, dès le 12 août de la même année, il annonce que le journal comptera bientôt six pages. "De cette façon, nous pourrons offrir à nos fidèles abonnés des matières plus variées et plus compactes, tout en donnant satisfaction à notre clientèle d'annonceurs, qui grandit de jour en jour ${ }^{30}$. " C'est chose faite le 26 août. Si le contenu rédactionnel est somme toute peu modifié, laissant la part belle aux nouvelles politiques, les publicités sont effectivement beaucoup plus nombreuses. Les marchands et professionnels locaux sont omniprésents et ils côtoient des

29 Ibid. Jusqu'au 4 octobre 1895, le cartouche du Franco-Canadien sera publié à la page 3; par la suite les deux noms seront réunis, et ce, durant plusieurs décennies. Le journal s'intitulera Le Canada français et Le Franco-Canadien.

30 "Au jour le jour ", Le Canada français et Le Franco-Canadien, 12 août 1898, p. 1. 
marchands montréalais, des compagnies de transport et des vendeurs de médicaments patentés.

Moins d'un an plus tard, deux nouvelles pages sont ajoutées au journal. Dans le bref article faisant état de cette modification, Gabriel Marchand n'est pas peu fier d'annoncer que son journal a su séduire de nombreux lecteurs et annonceurs et qu'il peut dès lors offrir encore plus de matière à lire à ses abonnés. Il profite de l'occasion pour souligner les progrès du goût de la lecture chez ses compatriotes et l'importance du rôle de la presse dans la société : «On a compris qu’il ne suffit plus, pour conserver sa place au soleil, de se faire mouvoir les bras, mais qu'il faut, par le facile et peu dispendieux intermédiaire de la presse, se mettre en état de disputer aux autres et d'arracher au besoin sa part légitime d'influence politique et sociale ${ }^{31}$. " Alors que la presse d'information se développe très rapidement, le journal demeure à ses yeux un important outil d'éducation et d'influence ${ }^{32}$.

Le 3 novembre 1899 apparaît pour la première fois dans le journal la chronique de Jean Rémuna, pseudonyme d'Arsène Bessette $^{33}$. Chaque semaine, le jeune journaliste, ami de Gabriel Marchand ${ }^{34}$, fait paraître un texte sur des sujets aussi variés que la grippe, le bonheur ou l'actualité du moment. Du 16 novembre 1906 au 16 juillet 1909, les lecteurs retrouveront ses textes dans une chronique intitulée "Modernités ». Bessette propose à son lectorat des réflexions sur différents sujets plus ou moins liés à l'actualitée ${ }^{35}$ ou

31 "Notre journal ", Le Canada français et Le Franco-Canadien, 30 juin 1899, p. 1.

32 Il ne faut d'ailleurs pas oublier que la presse d'information, sous des allures plus objectives, est encore largement utilisée comme moyen d'influencer les lecteurs.

33 Il abandonnera ce pseudonyme en juin 1901.

34 Avant son arrivée au Canada français, Arsène Bessette avait collaboré durant quelques mois à La Patrie de Montréal, mais c'est vraiment à l'hebdomadaire de Saint-Jean qu'il fait son apprentissage (voir Aurélien Boivin, "Bessette, Arsène ", dans $D B C$ en ligne, vol. 15, Québec, Université Laval; Toronto, Université de Toronto, 2005, [http://www.biographi.ca/fr/bio/bessette_arsene_15F.html].

35 Il utilise souvent un fait divers comme prétexte pour réfléchir sur un enjeu social plus général. Par exemple, un procès pour meurtre qui se déroule à New York devient l'occasion de discuter de la valeur d'un "plaidoyer de folie " ( Modernités ", Le Canada français, 14 février 1908, p. 2). 
des critiques littéraires ou artistiques. Friand de théâtre, un art particulièrement malmené par les élites cléricales, Bessette suit de près l'actualité théâtrale montréalaise, dont il prend régulièrement la défense ${ }^{36}$. Antiféministe, il critique souvent le tempérament trop " passionné » des femmes et il invite les jeunes filles à réfléchir au coût, parfois très élevé, de l'indépendance et de la liberté ${ }^{37}$. Son propos est parfois plus philosophique, encourageant, dans une envolée tout à fait libérale, la créativité et l'ouverture d'esprit :

En plein vingtième siècle, alors [que] le génie humain a inventé tant de merveilles, que la science a pénétré les secrets les plus précieux de la nature, l'obstacle formidable du préjugé, le mensonge des vieilles traditions maintiennent encore l'homme dans un état de demi-esclavage, l'empêchent de bénéficier de son savoir, de jouir enfin de la liberté dont s'honore aujourd'hui tout citoyen honnête et laborieux ${ }^{38}$.

À partir de juillet 1909, la chronique " Modernités " disparaît du journal, et Arsène Bessette ne signe plus que quelques rares textes. Cela ne signifie pas qu'il n'écrit plus dans le journal, mais que sa présence dans les pages de l'hebdomadaire est moins marquée ${ }^{39}$. Quoi qu'il en soit, les chroniques d'Arsène Bessette ont permis au Canada français de se doter d'une personnalité singulière. Le journal a alors quitté le champ quasi exclusif du commentaire politique pour s'ouvrir à des horizons beaucoup plus variés. Bessette mettra d'ailleurs cette expérience à profit dans son très controversé roman Le débutant, qui

36 Arsène Bessette, "Modernités ", Le Canada français, 5 mars 1909, p. 2.

37 Arsène Bessette, "Modernités ", Le Canada français, 5 juillet 1907, p. 2.

38 Arsène Bessette, "Modernités ", Le Canada français, 19 mars 1909, p. 2.

39 L'historiographie indique toujours que Bessette a collaboré régulièrement au Canada français jusqu'en 1917, mais un examen attentif du journal montre que cette collaboration n'est plus aussi évidente à partir de 1909. Même si l'on retrouve encore quelques textes signés de sa main (septembre 1910, novembre 1911, avril 1913), Bessette a peut-être utilisé un autre pseudonyme. Le nom de Bessette n'apparaît dans les répertoires Lovell de Montréal qu’en 1917. Il n’aurait donc pas habité Montréal avant cette date. Une recherche exhaustive serait essentielle pour bien comprendre le parcours de cet auteur. 
paraîtra en 1914. Ces chroniques ne sont pas ancrées dans un cadre régional : elles témoignent de l'action médiatique d'un auteur qui joue un rôle de passeur entre la région et la métropole.

\section{Le virage vers la presse d'information}

En juin 1908, Gabriel Marchand est élu député libéral de la circonscription de Saint-Jean. Il cède alors la propriété du journal à la Compagnie de publication du Canada français, mais il en demeure l'éditeur jusqu'à son décès, survenu subitement le 16 septembre $1910^{40}$. Le 30 décembre de la même année, on avise les lecteurs que les ateliers de production déménageront dans un nouvel édifice et qu'il est possible que le journal ne soit pas publié la semaine suivante. On sollicite leur patience : "L'amélioration de notre journal les récompensera de leur indulgence par la suite ${ }^{41}$. » Le 27 janvier 1911, on annonce, en effet, un changement majeur : dès la prochaine livraison, le journal comptera seize pages, plutôt que huit, et il sera illustré. Il est plausible de croire que les nouveaux propriétaireséditeurs se sentent obligés de réagir face à la présence des grands quotidiens d'information montréalais sur le marché de Saint-Jean. La concurrence est féroce; il faut donc l'affronter. Afin de conquérir de nouveaux lecteurs, le journal lance un grand concours de popularité et d'abonnement au cours duquel plus de 1600 cadeaux certains de grande valeur, comme des pianos ou des montres en or - seront distribués ${ }^{42}$.

La métamorphose du journal est importante. La page éditoriale, qu'on retrouve à la page 8 , conserve toujours son lien avec le Parti libéral, mais le reste de l'hebdomadaire n'est plus construit autour

${ }^{40}$ Ronald Martel, Histoire de la presse hebdomadaire au Québec : Estrie, Montérégie, Montréal, Hebdos Québec, [2009?], p. 47. Ce changement de propriétaire constitue certainement une piste à suivre pour mieux comprendre les relations entre Arsène Bessette et le journal.

41 "Avis à nos lecteurs ", Le Canada français et Le Franco-Canadien, 30 décembre 1910, p. 1. Le journal a finalement paru le 6 janvier 1911.

42 Le Canada français et Le Franco-Canadien, 3 février 1911, p. 4. 


\section{Le Canada Francais}

Et LE FKANC( ).CANDIEN

\section{AVIS AUX AGENTS}

Vu notre grand concours nous drvoas suspendre toute commis. sion aux agents pour l'obtention et la collection des abonnements ¿D'un autre coté, nous leur donnons dans notre concours des avantages bien plus considera. bles qua par le passé. Que chacun en profite.

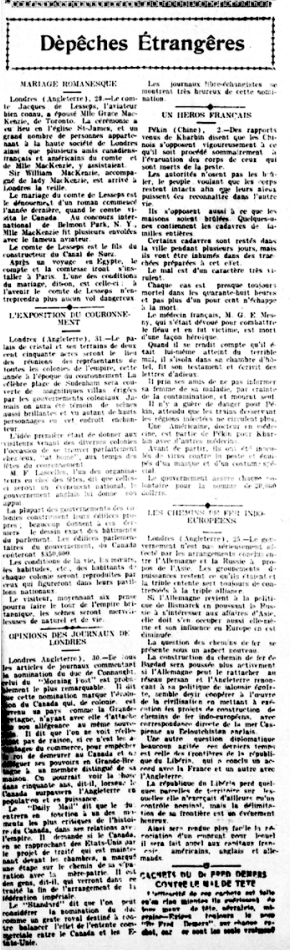

\section{Grand Concours du Canada Français}

Voyez-en les détails dans nos pages intérieures.

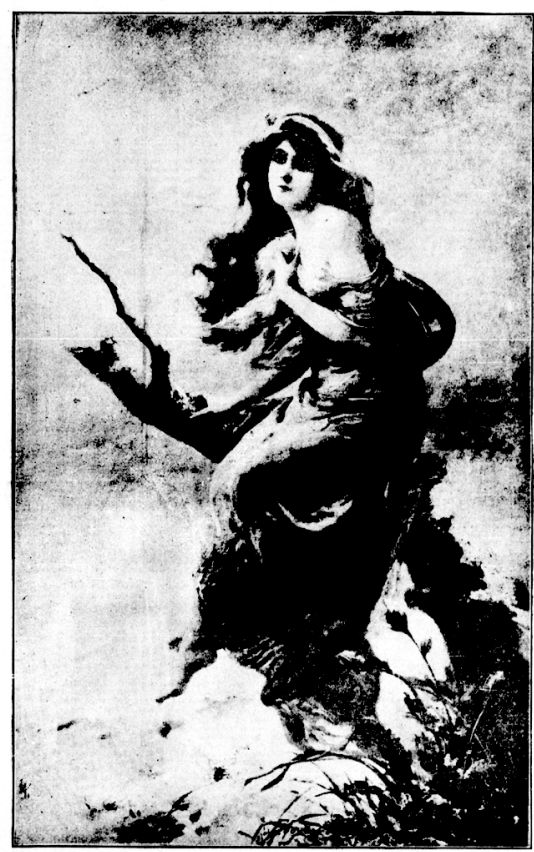

\section{LA CIGALE}

Notre Prime Photogravure No. 1

$$
\text { GRANDECR 20 } 2 \text { 98 POCCRS }
$$

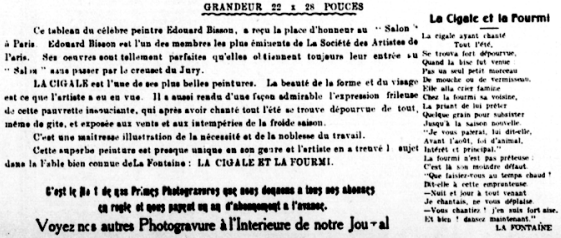

Figure 2 - Le Canada français et Le Franco-Canadien, 3 février 1911, p. 1. Source: Bibliothèque et Archives nationales du Québec, [En ligne], [http://numerique. banq.qc.ca/patrimoine/details/52327/1773707]. 
de cette unique motivation de faire valoir le point de vue du Parti. Le journal d'opinion semble vouloir céder la place au journal d'information. À l'image des grands médias de masse, il est beaucoup mieux structuré : à côté des dépêches internationales, du "Courrier de Paris » et des « Nouvelles des États-Unis », qui constituent le cœur des nouvelles internationales et qui sont présentées dès les premières pages, on retrouve la chronique " $\mathrm{A}$ travers le Canada », les « Nouvelles de St-Jean » et les "Correspondances de la campagne ». Les nouvelles locales occupent une page entière. On y retrouve pêle-mêle des mondanités, des faits divers, des informations sur la vie politique municipale et quelques nouvelles économiques. Les « correspondances de la campagne " sont composées selon le modèle établi dans la presse d'information : classées selon les municipalités plus ou moins limitrophes à Saint-Jean, elles proposent des brèves qui relatent essentiellement des mondanités et des faits divers locaux. Le journal manifeste toujours de l'intérêt pour le commerce et l'agriculture, et cette chronique, considérablement enrichie, occupe une page entière. Elle informe les lecteurs sur le cours des denrées et la qualité des récoltes, mais elle prodigue aussi quelques conseils en matière d'agriculture. De nouvelles sections cherchent à séduire un lectorat plus varié : on cible toute la famille avec une page "Mode pratique" pour les femmes, et une "Page amusante » offre des bandes dessinées aux enfants. Les annonces publicitaires sont nombreuses, mais elles sont loin d'occuper une part aussi importante du contenu que dans les quotidiens nationaux : des commerçants et des hommes d'affaires locaux partagent les pages de l'hebdomadaire avec des commerçants montréalais et des publicités nationales. Bref, on reprend ici la recette qui a fait le succès de la grande presse d'information.

L'expérience ne fait cependant pas long feu puisque dès le mois de décembre de la même année, le journal, quoique toujours illustré, ne compte plus que douze pages. À la fin de la guerre, en 1918, il est revenu à un format de huit pages, les illustrations ont disparu à l'exception des annonces publicitaires - et l'éditorial a réintégré la une. Les importants trous dans la collection durant la période de la 
guerre ne permettent cependant pas de connaître le moment précis de ce retour à un format plus modeste. Il est toutefois permis de croire que les restrictions sur le papier et l'encre durant la guerre peuvent avoir été un facteur important dans ce changement, mais la perte de revenus publicitaires devrait aussi être prise en considération.

Le 31 mai 1918, à l'occasion du 58 e anniversaire du journal, la direction réitère sa volonté de poursuivre courageusement la mission qui avait été celle des fondateurs.

[D] evant la situation qui est faite à la presse, il faut être lesté d'une forte dose de courage pour entreprendre de continuer la tâche de renseigner le public et guider l'opinion. Ce courage nous l'avons; notre vaillance ne s'est pas émoussée dans la rudesse des combats antérieurs et nous sommes toujours disposé à affronter les luttes futures ${ }^{43}$.

La Première Guerre n'étant pas encore terminée, on peut croire que la censure alors exercée sur les journaux fait partie de cette « situation faite à la presse ${ }^{44}$ ". Il est aussi intéressant de noter que Le Canada français affiche toujours sa volonté très affirmée de guider l'opinion ; le journal ne se contente pas d'être un véhicule d'informations régionales. Cependant, force est d'admettre que la situation n'était pas temporaire, donc pas uniquement liée au contexte de la guerre: jusqu'au 30 août 1934, le nombre de pages du journal oscille entre six et huit par numéro.

À ce moment-là, un nouveau coup de barre est donné. En rupture avec la pratique habituelle du journal, et parce que la direction se dit sensible au goût des lecteurs, on adopte le format tabloïd afin de rendre le journal plus attrayant et plus facile à lire : «Le nouveau format tabloïd tend à se répandre de plus en plus et les nombreuses

43 "Notre 58 anniversaire ", Le Canada français et Le Franco-Canadien, 31 mai 1918 , p. 1.

${ }^{44}$ Voir Myriam Levert, «Le Québec sous le règne d'Anastasie : l'expérience censoriale durant la Première Guerre mondiale ", Revue d'histoire de l'Amérique française, vol. 57, $\mathrm{n}^{\circ} 3$ (hiver 2004), p. 333-364. 


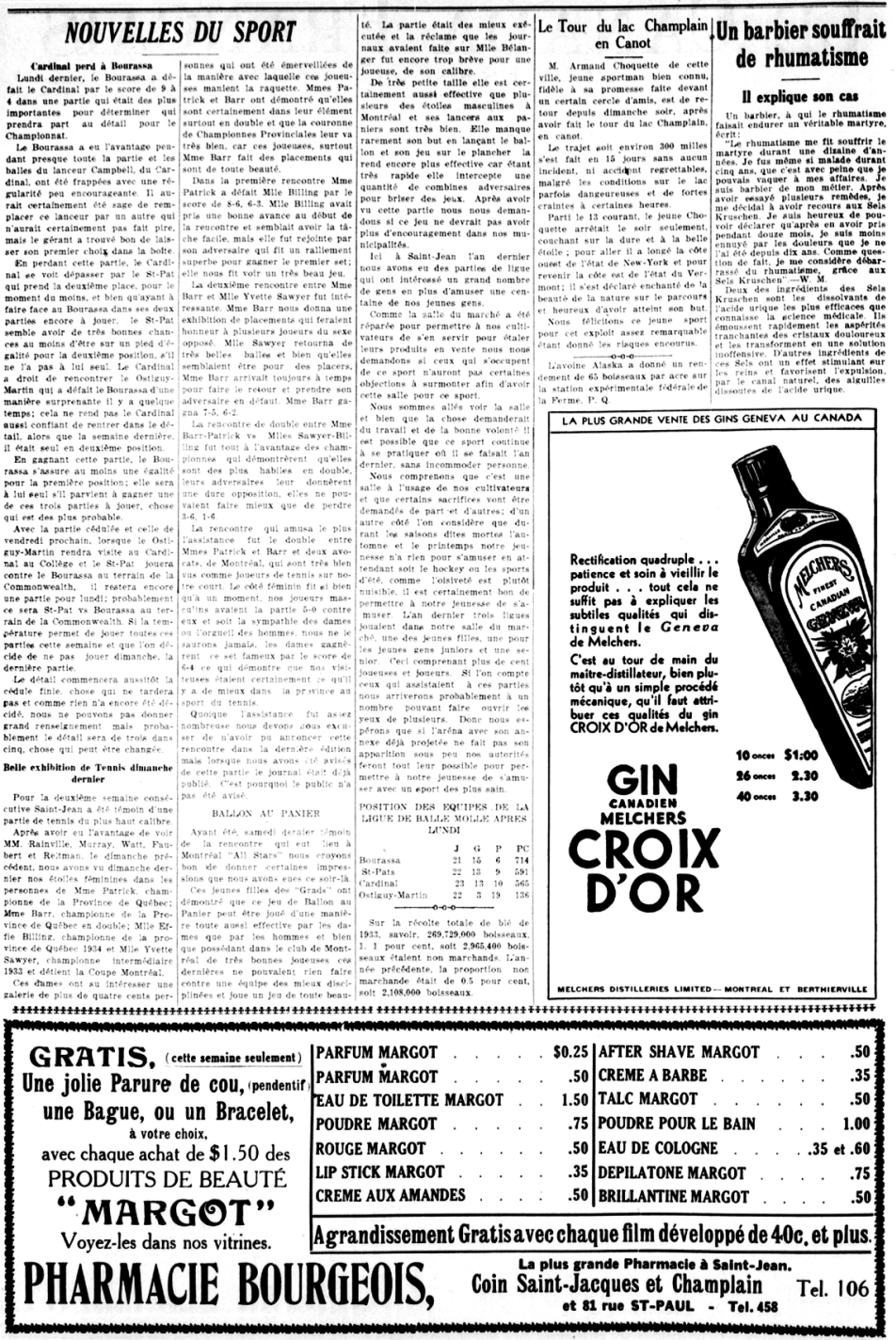

Figure 3 - Le Canada français et Le Franco-Canadien, 30 août 1934, p. 2. Source : Bibliothèque et Archives nationales du Québec, [En ligne], [http://numerique. banq.qc.ca/patrimoine/details/52327/1774686]. 
appréciations entendues à l'adresse des journaux et des revues de ce genre nous ont incités à l'adopter ${ }^{45}$. " L'arrivée du Petit Journal (1926) et de L'Illustration (1930) sur le grand marché montréalais, deux journaux de format tabloïd qui optent pour une formule beaucoup plus populaire, n'est certainement pas étrangère à cet important virage. Le contenu et la mise en page du Canada français restent sensiblement les mêmes, mais un ajout non négligeable est apporté : une section intitulée " Nouvelles du sport » est insérée à la page deux. Les équipes et les athlètes locaux sont alors à l'honneur. L'éditorial paraît à la dernière page, juste à côté des petites annonces.

En juillet 1937, un magazine est ajouté au journal. Cette section, qui a sa propre une, propose un contenu très diversifié qui se distingue clairement de la portion centrée sur les actualités. Nouvelles littéraires, chroniques historiques, bandes dessinées, " actualité par l'image " composent ce supplément illustré de huit pages qui se veut à la fois instructif et divertissant, mais nullement centré sur des intérêts régionaux. Les éditeurs testent ainsi une formule visant à concurrencer les revues généralistes et les suppléments hebdomadaires des quotidiens. Pour bien marquer la volonté de l'éditeur de plaire aux lecteurs, on invite ces derniers à soumettre toutes suggestions qui pourraient améliorer le magazine.

En novembre de la même année, le cartouche d'entête est renouvelé et, dans un style plus travaillé, les deux titres du journal sont dorénavant superposés, Le Canada français étant mis en évidence. La direction est alors fière d'annoncer que ce nouvel entête est l'œuvre d'un concitoyen de Saint-Jean, artiste autodidacte ${ }^{46}$. Le cartouche expose aussi sa nouvelle devise : "Hebdomadaire dévoué aux intérêts de toute la Région ». On abandonne donc le lien avec le Parti libéral et l'on met l'accent sur la volonté de satisfaire les intérêts régionaux.

45 "Notre journal et son nouveau format tabloïd ", Le Canada français et Le FrancoCanadien, 30 août 1934 , p. 1.

46 "Notre nouvel en-tête ", Le Canada français et Le Franco-Canadien, 25 novembre 1937 , p. 4. 


\section{Le Canada Francais \\ Et LE FRANCO-CANADIEN}

SECTION MAGAZINE
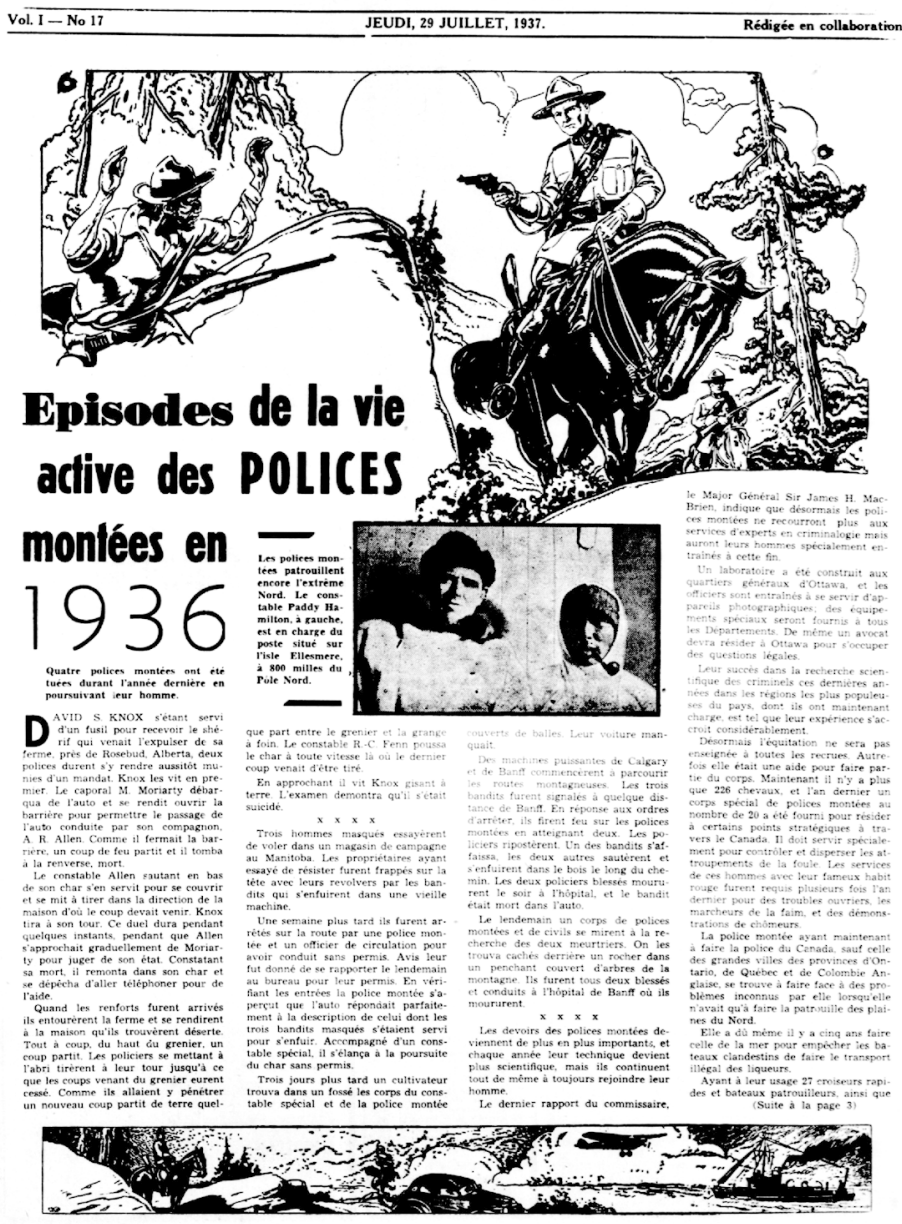

- SUPPLEMENT

- Page

Figure 4 - Le Canada français et Le Franco-Canadien, 29 juillet 1937, p. 1. Source : Bibliothèque et Archives nationales du Québec, [En ligne], [http://numerique. banq.qc.ca/patrimoine/details/52327/1774857]. 
Durant près de trente ans, le nombre de pages du journal augmentera peu à peu, mais on ne modifiera plus de manière notable sa facture, si ce n'est une composition plus aérée de la une. Cependant, l'accent sera de plus en plus mis sur les nouvelles locales et régionales. On semble avoir trouvé la formule qui convient aux lecteurs et aux annonceurs. La facture demeurera assez similaire jusque dans les années 1970.

L'évolution qui caractérise la pratique journalistique de cet hebdomadaire régional met en lumière les difficultés rencontrées par les éditeurs qui doivent concurrencer les quotidiens nationaux. Saint-Jean fait partie de la zone de distribution de La Presse et de La Patrie, les deux quotidiens montréalais francophones les plus populaires et, s'il veut survivre, Le Canada français doit trouver sa niche. L'apparition des tabloïds à la fin des années 1920 crée une nouvelle tension, un nouvel horizon auquel il faut se référer. Le système dans lequel évolue Le Canada français est en constante transformation, et l'hebdomadaire régional ne peut l'ignorer. D'ailleurs, les nombreuses références aux grands journaux, qui constituent une sorte d'étalon de mesure, témoignent de l'attention constante portée sur eux. Mais cette entreprise de presse n'ayant pas les moyens de s'offrir des mises en page très sophistiquées, on opte donc pour une certaine simplicité, tout en mettant le cap sur la proximité avec le lecteur : ce journal est son journal.

Il serait donc possible de diviser l'histoire du Canada français en trois périodes. On pourrait aisément qualifier la première, sous la gouverne des Marchand, père et fils, jusqu'à 1908, de l'ère politique : les enjeux politiques fédéraux, mais surtout provinciaux sont au cœur de la mission et du contenu du journal. Ce dernier propose à ses lecteurs une mise en page modifiée, mais il demeure essentiellement un journal d'opinion politique. Il met en scène sa proposition régionale essentiellement par les annonces publicitaires et quelques nouvelles locales. La seconde période s’étire jusqu'aux années 1930 ; elle est marquée par l'évolution de la pratique journalistique, le passage d'une presse d'opinion essentiellement politique à une presse d'information. Malgré le fait que les liens du journal avec le Parti libéral 
soient encore assez solides - du moins jusqu'en 1937 -, on met de plus en plus l'accent sur le développement économique et industriel, on veut vendre le potentiel de la région et souligner à grands traits son dynamisme. On peut croire que, d'une part, on souhaite attirer investisseurs et nouveaux résidents, et, d'autre part, que l'on veut limiter l'attraction que la ville de Montréal exerce sur la population de la région. Une troisième période, qui s'ouvre sur le passage au format tabloïd, montre que le journal, tout en étant sensible à l'évolution du goût des lecteurs, cherche à témoigner de la spécificité socioculturelle de la ville et de son environnement. Il ne peut pas faire la lutte aux grands quotidiens montréalais, mais il a trouvé sa niche et il devient le relais essentiel de la vie régionale.

Cette hypothèse, selon laquelle l'histoire du Canada français peut être divisée en trois périodes, permet de mettre en perspective l'évolution d'un journal, le milieu d'où il émane et le système médiatique dans lequel il s'insère. Si cette affirmation est valable pour les grands quotidiens ${ }^{47}$, elle l'est encore plus pour un journal régional.

\section{Conclusion}

Un autre volet de la vie de ce journal pourrait s'inscrire dans un cadre d'analyse qui s'intéresse à la sociologie des journalistes et du lectorat. Qui sont ceux qui ont écrit dans ce journal? Viennent-ils de la région? À leurs yeux, le travail dans un journal régional a-t-il la même valeur que celui effectué dans un quotidien national? On sait qu'au XIX ${ }^{\mathrm{e}}$ siècle, Félix-Gabriel Marchand était le rédacteur principal du Franco-Canadien et, parce qu'il a été premier ministre du Québec, on le connaît assez bien. Il a essentiellement utilisé le journal à des fins politiques, et cela transparaît abondamment durant la période

${ }^{47}$ Comme l'ont montré de nombreux chercheurs à partir de la théorie de Maurice Mouillaud, "Le système des journaux (théorie et méthodes pour l'analyse de presse) ", Langages, $3^{\mathrm{e}}$ année, $\mathrm{n}^{\circ} 11$ (septembre 1968), p. 61-83. Voir aussi Marquis, Un quotidien pour l'Église. 
où il en est le directeur. En juin 1898, son fils Gabriel prend la relève : il affirme alors répondre à des pressions politiques, mais il souhaite néanmoins changer le visage du journalisme en refusant de jouer au jeu des injures. Il promet une "critique courtoise et vigoureuse, arme favorite des gentilshommes ${ }^{48}$ ", mais son journal conserve néanmoins les caractéristiques de la presse d'opinion. Marchand fils a su s'entourer de rédacteurs talentueux, dont Arsène Bessette, que nous avons déjà présenté.

Les autres collaborateurs du journal, éditeurs ou rédacteurs, sont moins connus. Un travail colossal attend le chercheur qui voudrait faire la prosopographie du personnel de presse, puisque les outils habituellement utilisés par les chercheurs, comme les annuaires ou les dictionnaires biographiques, se sont peu intéressés à ces artisans. On y retrouve certes quelques têtes d'affiche du journalisme canadienfrançais, mais des individus comme Louis-Omer Perrier, qui a été à la barre du Canada français de 1918 à 1958 et qui a été candidat libéral défait aux élections provinciales de 1941 et de 1944, mériteraient qu'on analyse en profondeur leur contribution. Une compréhension globale de la presse régionale ne peut faire l'économie de cette recherche biographique.

En conclusion, l'exemple du Canada français de Saint-Jean nous montre à quel point l'étude de la presse régionale québécoise peut être stimulante. Corpus qui se distingue nettement de la presse quotidienne des grands centres urbains, les journaux régionaux nous plongent au cœur de réalités multiformes. Bien qu'ils ne puissent concurrencer les grands quotidiens, ces journaux répondent à différents besoins - informations sur les enjeux régionaux, publicités pour les gens d'affaires locaux, quête d'une identité régionale propre tout en ne négligeant pas la mission initiale de la presse d'opinion du XIX ${ }^{\mathrm{e}}$ siècle, éduquer et éclairer l'opinion publique. Les journaux régionaux québécois ont indéniablement participé à la constitution

48 Gabriel Marchand, "Au public ", Le Canada français, 17 juin 1898, p. 1. 
de la culture médiatique d'une grande partie de la population; leur histoire n'est peut-être pas encore suffisamment développée, mais les sources de réflexion sont multiples. 